\title{
Electromagnetic Freezing in a Widespread Frequency Range of Alternating Magnetic Fields
}

\author{
A. C. Rodríguez ${ }^{1,2} \cdot$ L. Otero $^{1} \cdot$ J. A. $\operatorname{Cobos}^{2} \cdot$ P. D. Sanz ${ }^{1}$ (D)
}

\begin{abstract}
The effectiveness of electromagnetic (EM) freezing in improving freezing kinetics and/or the quality of frozen foods constitutes a current controversial topic in Food Technology. The dipolar nature of water could explain potential effects of electric fields on freezing, but the physical basis that supports effects of magnetic fields on freezing is not clear. Therefore, it is in some way striking that the only EM freezers existing at the market are those that generate magnetic, and not electric, fields to assist the freezing process. In this paper, a comprehensive review of the state of the art in EM freezing is presented. The results reported in the literature on the effects of both static and oscillating, either electric or magnetic, fields on supercooling and freezing kinetics are controversial, even for the simplest system, that is, pure water. Moreover, the reviewed results show that frequency and dielectric relaxation could play an important role on water supercooling. Thus, positive effects on freezing have been found in experiments with oscillating, both electric and magnetic, fields of frequencies significantly higher than that of the mains. As oscillating electric fields are induced in the presence of oscillating magnetic fields, this opens a door to explain the potential effects of oscillating magnetic fields on freezing. For a correct interpretation of the data, future research should consider any induced field during the freezing experiments and its potential consequences. All the above reveals the urgent need to perform high-quality scientific research and well-designed experiments, at wide field strengths and frequencies, that can be replicated and confirmed by different laboratories.
\end{abstract}

Keywords Electromagnetic freezing $\cdot$ Electric field $\cdot$ Magnetic field $\cdot$ Freezing kinetics

\section{Introduction}

One of the biggest current challenges in bioscience and food technology is the achievement of freezing processes suitable for preservation of materials for long periods of time with no

\footnotetext{
A. C. Rodríguez

antonio.c.rguez@gmail.com

L. Otero

1.otero@ictan.csic.es

J. A. Cobos

ja.cobos@upm.es

P. D. Sanz

pedro.sanz@csic.es

1 Instituto de Ciencia y Tecnología de Alimentos y Nutrición (ICTAN CSIC), c/ José Antonio Novais, 10, 28040 Madrid, Spain

2 Centro de Electrónica Industrial, Universidad Politécnica de Madrid (CEI UPM), c/ José Gutiérrez Abascal, 2, 28006 Madrid, Spain
}

or negligible loss of quality. In this connection and looking for safety, quality, economy, or other reasons, important endeavors have been performed by scientists and technicians during the last decades to improve freezing processes. Current research is focused on increasing both the ice nucleation rate and the speed of heat removal from the system. The ultimate aim is to generate a large number of small ice crystals, evenly distributed throughout the product, that produce negligible damage on it. Nowadays, several physical magnitudes as ultrasounds, high pressure, or electromagnetic fields (EMFs) are being studied to assess their ability to affect ice nucleation. Among them, only EMFs have been already implemented in commercial freezing systems.

In the last years, Woo and Mujumdar [61]; Xanthakis et al. [63]; and Otero et al. [37] have reviewed a large variety of electromagnetic (EM) freezing systems, paying attention to their relevance for this purpose in biological models, cells, tissues, alive organisms, and food. What is inferred from these review articles, given the discrepancies found in scientific papers, is that neither the physical action of EMFs on ice 
formation nor the potential improvement in the quality of the frozen product has yet been totally clarified.

Water is a dipolar molecule and, therefore, when subjected to an electric field (EF), molecules are oriented, lining up their molecular dipoles along the direction of the field. This wellknown effect could be a solid starting point to explain the effects of EFs on water freezing. However, things get a little more complicated when looking for arguments to explain the potential effects of magnetic fields (MFs) on water freezing. Water has no net magnetic moment. When subjected to an external MF, the orbital motion of electrons is altered in such a way that the induced magnetic moments oppose the external field and water is, therefore, a diamagnetic substance. The magnetic susceptibility of water, that is, its magnetic response to the applied MF, is rather low $\left(\chi_{v}=-9.035 \times 10^{6}\right)$ and, therefore, little effects of weak MFs must be expected on water.

It is, therefore, in some way striking that, nowadays, the only electromagnetic freezers existing at the market are those that generate magnetic fields, and not EFs, to assist the freezing process. This has happened even though the effectivity of MFs in improving freezing has not yet been proved. Several companies have patented and/or marketed freezers that apply different types of MFs to tentatively improve the quality of frozen food. Such is the case of ABI Co., Ltd. (Chiba, Japan) that sells "CAS (Cells Alive System) freezers" that combine static and oscillating MFs. On the other hand, Ryoho Freeze Systems Co., Ltd. (Nara, Japan) commercializes "Proton freezers" that use static MFs and EM waves $[1,50]$. These innovative freezers actually consist of a MF generator attached to a conventional quick-freezing unit. Additionally, in Proton freezers, an EM wave generator is also added. Depending on the application, the MF or the EM waves may be ceased after certain "supercooling period" to quickly induce the immediate freezing of the product, or they may be kept throughout the whole preservation period for a better control of the crystallization mechanism [41]. Many videos that publicize the benefits of both CAS and Proton freezers can be found on the Internet [37]. According to commercial advertisements, both CAS and Proton freezers are able to generate tiny ice crystals throughout the whole frozen product. Besides, they are claimed to prevent cell destruction and preserve the quality of fresh products intact after thawing $[1,17]$. Consequently, many food processors, restaurants, hotels, hospitals, and research centers, not only in Japan but also all over the world [19, 23], have acquired these magnetic freezers. Different food products such as fish (tuna, salmon, or cod, among others), sea urchin, shrimps, sashimi grade seafood and sushi, meat (Wagyu beef, Sufflok lamb, or duck, for example), foie gras, fruits (green mango, lychee, and so on), delicate doughs, and milk products are nowadays magnetically frozen and commercialized as superior-quality food products. Freezing temperatures in magnetic freezers are usually very low $\left(-60^{\circ} \mathrm{C}\right.$ and lower) and many doubts exist about the real responsible for the improved results: these low temperatures or the magnetic fields applied.

Freezers with coupled EFs have also been developed but only at lab-scale. Perhaps the multiple difficulties involved in implementing EFs at industrial scale are the reason of the lack of this type of commercial freezers. Phenomena such as sparks, electric arcs, and corona discharges must be avoided and this implies restrictions on the voltage applied, the distance between electrodes, and their characteristics. The electrodes should have preferably parallel surfaces and they must be sufficiently polished to prevent the rise of charge density which can appear at protruding parts. Moreover, rough points can enhance corona discharges, that is, the ionization of air around the charged electrode surfaces [10]. According to Dalvi-Isfahan et al. [9], the distance between electrodes should be smaller than their diameter. Thus, in EF-freezing scientific works, electrode separation ranges between $0.3 \mathrm{~mm}$ [15] and $20 \mathrm{~mm}$ [60]. This small distance obviously limits the dimensions of the products to be frozen. For the EF generation, the electrode plates can be immersed directly into the product $[14,15,43]$ or they can be positioned external to the sample. In this case, to avoid current across the product, the sample should not be in contact with the electrodes, so the practical distance is still more reduced. In many cases, high voltages, in the order of $\mathrm{kV}$, are employed and the condensation of water or the deposit of frost on the surfaces between parts which should be isolated could cause safety problems. Thus, in industrial designs, the circuit generating the EF should be provided with a switching device to cut off the high voltage supply when users open the freezer door [24]. Moreover, users should be prevented from touching surfaces at different potential to avoid any electrical risk. All these problems and technical difficulties could be the cause of the current lack of commercial EF freezers.

This paper presents a comprehensive review of the state of the art in electromagnetic freezing. Even though current commercial equipment uses both static and oscillating MF generators and EM waves, in the present paper, we will leave aside the employment of EM waves and only focus on MFs. Moreover, as varying electric and magnetic fields are closely linked, static and oscillating EFs are also presented. Special attention is paid to the frequency of oscillating fields which could play a main role in improving freezing kinetics.

\section{The Physical Basis of Electromagnetic Freezing}

Freezing is a well-known process that takes place in three key steps: precooling, phase transition, and tempering. Freezing kinetics strongly affects the size and shape of the ice crystals formed and, consequently, the quality of the frozen products. As stated in the introduction, the ultimate aim is to generate 
small ice crystals, evenly distributed throughout the product, that produce negligible damage on it. A proved strategy to generate small ice crystals is to induce large supercooling in the product to be frozen. In fact, the interest of applying electric and/or magnetic fields during freezing just lies in their potential to maintain the product unfrozen, that is, in a supercooled state at a temperature below its freezing point. It is well-known that the larger the degree of supercooling (difference between freezing and nucleation temperatures), the larger the amount of ice instantaneously formed at nucleation. Thus, as the nucleation temperature descends, the ice nucleation rate increases around tenfold per each ${ }^{\circ} \mathrm{C}$ [7] and, consequently, the phase transition time is reduced. Both high nucleation rates and short phase transition times are key requirements to obtain small and uniformly distributed ice crystals throughout the whole volume of the frozen product [65]. It is admitted that small ice crystals reduce cellular damage and quality losses in frozen products $[45,65]$. So, if the application of EMFs during freezing was effective in increasing supercooling, injury produced in electromagnetically frozen products would be reduced compared to that caused by traditional methods.

The physical basis of electric field-assisted freezing seems to be much clearer than that of magnetic field-assisted freezing. The dipolar character of water can explain why EFs can align water molecules in the direction of the field. Theoretically, static electric fields (SEFs) can strengthen hydrogen bonds parallel to the field [58] and this would reduce the extent of supercooling needed to initiate nucleation. Consequently, fewer ice nuclei would be instantaneously formed and longer phase transition times and largest ice crystals should be expected. Even though some specific processes such as lyophilization can find benefits from large ice crystals, in general terms, SEFs would have negative consequences on most freezing applications. However, when oscillating electric fields (OEFs) are applied, water molecules are continuously vibrating to align the continuously changing direction of the field. This continuous vibration will hinder ice nucleation and, therefore, large degrees of supercooling and their associated benefits could be attained.

Now, the remaining question is: how can MFs affect ice nucleation? The published patents claim that the application of MFs during freezing causes a reorientation of the electronic and nuclear spins of the water molecules in the sample to be frozen [41]. It is argued that the application of MFs induces forces of magnetic vibration in the water molecules, which inhibits the early formation of ice crystals and their growth even at very low temperatures. Consequently, water crystallization of the food could be deferred and a high degree of supercooling attained [12]. However, none of these arguments have been scientifically proved. As established in the introduction, water is a diamagnetic substance of low magnetic susceptibility and, therefore, weak MFs, as those usually employed in commercial MFfreezing systems, should have little effect on water molecules.
Many authors in the literature have performed different experiments to tentatively show the potential effects of electric and magnetic fields on freezing. However, as stressed by Otero et al. [37], many obstacles associated with the freezing process hamper the experimental work. Among them, the stochastic nature of the heterogeneous nucleation of supercooled water is, with no doubt, one of the main causes of the controversial results frequently found in the literature. Even when all the factors involved in the tendency of a system to supercool (sample size, impurities, type and surface of the container, temperature, cooling rate, and so on) are carefully controlled, different degrees of supercooling occur in repeated experiments. This makes it necessary to perform an adequate experimental design, with enough number of repeated experiments, and a rigorous statistical analysis before drawing valid conclusions. However, in many published papers, neither the experimental design nor the statistical analysis is presented and this casts doubts on the validity of the reported results. Moreover, when freezing experiments are performed in biomaterials, such as food or biological specimens, their inherent variability also hampers the reproducibility and replicability of the experimental conditions and, therefore, this variability must be considered before drawing premature conclusions. Finally, other difficulties directly associated to electric and magnetic fields must also be taken into account. Thus, some factors such as impurities in the sample or spatial electric and/ or magnetic gradients throughout the product should not be neglected [37, 39].

\section{Application of Static Electric and Magnetic Fields for Freezing}

Static fields are defined by the field strength and, therefore, when searching on the effects of static fields on freezing, critical strength values, able to affect supercooling, are sought. However, the results reported in the literature for both SEFs and static magnetic fields (SMFs) are controversial and, sometimes, they do not apparently agree with the expected physics.

According to theory, Wei et al. [60] observed a rise of $1.6^{\circ} \mathrm{C}$ in the nucleation temperature of water when freezing under a $10^{5}-\mathrm{V} / \mathrm{m}$ SEF. However, they recognized that this SEF strength was lower than $1.5 \cdot 10^{7} \mathrm{~V} / \mathrm{m}$, the critical value predicted by molecular dynamics simulation to induce significant effects on the nucleation of pure water [53, 57, 58]. Hozumi et al. [15] and Hozumi et al. [14] also observed an increase in the nucleation temperature when they froze pure water while applying SEF strengths lower than that critical value, namely, $1.7 \cdot 10^{5}$ and 4 . $10^{5} \mathrm{~V} / \mathrm{m}$. On the contrary, Stan et al. [54] did not find any significant effect on the nucleation temperature of pure water when frozen under a $1.6 \cdot 10^{5} \mathrm{-V} / \mathrm{m}$ SEF. Another more puzzling discovery is reported by Xanthakis et al. [62] who froze pork tenderloin subjected to a $6 \cdot 10^{6}-\mathrm{V} / \mathrm{m}$ SEF. Even though, 
according to theory, the applied SEF reduced supercooling previous to ice nucleation, the ice crystals formed were significantly smaller than those without SEF. Accordingly, Dalvi-Isfahan et al. [9], working with even a weaker SEF $\left(5.8 \cdot 10^{4} \mathrm{~V} / \mathrm{m}\right)$, obtained an improvement in the quality of frozen lamb meat. Similarly, Jia et al. [21] applied several SEFs, of up to 2.5 . $10^{5} \mathrm{~V} / \mathrm{m}$, during the freezing of pork tenderloin and found a shorter phase transition time at $1.0 \cdot 10^{5} \mathrm{~V} / \mathrm{m}$. At this condition, $\mathrm{pH}$ of the samples was not significantly altered and color changes were smaller than those observed in conventionally frozen controls. Therefore, the authors concluded that SEF applied during freezing improved food quality.

Concerning SMFs, there exist a number of papers in the literature that analyze their effect on freezing kinetics of systems of different complexity, from pure water to real foods and biological specimens. The range of SMF strengths employed in these studies covers four orders of magnitude, from decimals to hundreds of $\mathrm{mT}$ (Fig. 1). However, no clear conclusions can be drawn from these studies because results are apparently contradictory, even for the simplest system, that is, pure water.

Thus, Zhou et al. [68] froze water under SMFs at relatively low strengths, up to $5.95 \mathrm{mT}$, and noticed that supercooling increased with the SMF strength. In contrast, Aleksandrov et al. [2], who worked with considerably stronger SMFs (71$505 \mathrm{mT}$ ), noted a reduction in water supercooling when increasing the SMF strength. On the other hand, Zhao et al. [66] did not detect any SMF effect (0-43.5 mT) either on supercooling or on the phase transition time for deionized water. Accordingly, Otero et al. [39] froze $10 \mathrm{~mL}$ pure water samples between two magnets, either in attractive (107-359 mT) or in repulsive $(0-241 \mathrm{mT})$ position, and found no effect of the SMF on the time at which nucleation occurred, the extent of supercooling, and the phase transition and total freezing times.

Some authors claim that, even though relatively weak SMFs have no direct effects on freezing kinetics of pure water, these SMFs can still affect freezing of food and biological products by altering other product components and/or their interactions with water molecules. Thus, unlike how it occurred in pure water, Zhao et al. [66] reported that SMFs of up to $43.5 \mathrm{mT}$ increased the nucleation temperature of $5 \%$ ethylene glycol solutions. The authors attributed this effect to a reinforcement of the hydrogen bonds of the ethylene glycol molecules. Moreover, they observed that SMFs increased supercooling and shortened the phase transition time of $5 \mathrm{~mL} 0.9 \% \mathrm{NaCl}$ solutions, which they ascribed to an enhanced mobility of $\mathrm{Na}^{+}$and $\mathrm{Cl}$ ions. Nevertheless, their results for $\mathrm{NaCl}$ solutions differ in part from those given by Mok et al. [33] who froze $2 \mathrm{~mL} 0.9 \% \mathrm{NaCl}$ samples disposed between two neodymium magnets. These authors introduced the direction of the MF forces as a new parameter which might play a relevant role in the freezing process. Depending on the magnets arrangement, the phase transition time increased by 17\% (480 mT, unlike magnet poles faced each other: attractive position) or reduced by $32 \%$ (50 $\mathrm{mT}$, like magnet poles faced each other: repulsive position) compared to the control. Nevertheless, in an attempt to reproduce such results, Otero et al. [39] froze $0.9 \% \mathrm{NaCl}$ solutions between two magnets in attractive (107-359 $\mathrm{mT})$ or in repulsive $(0-241 \mathrm{mT})$ positions and found no effects of SMFs on freezing kinetics.

Studies on the effect of SMFs on freezing kinetics of complex systems are especially scarce. Lou et al. [30] froze carps with very low SMFs $(0.36,0.72$, and $1.08 \mathrm{mT})$ and,
Fig. 1 Static and oscillating magnetic field strengths and frequencies employed in patents and scientific works on magnetic freezing. Red: patents; blue: papers on water and model systems; green: papers on food; orange: papers on biological materials

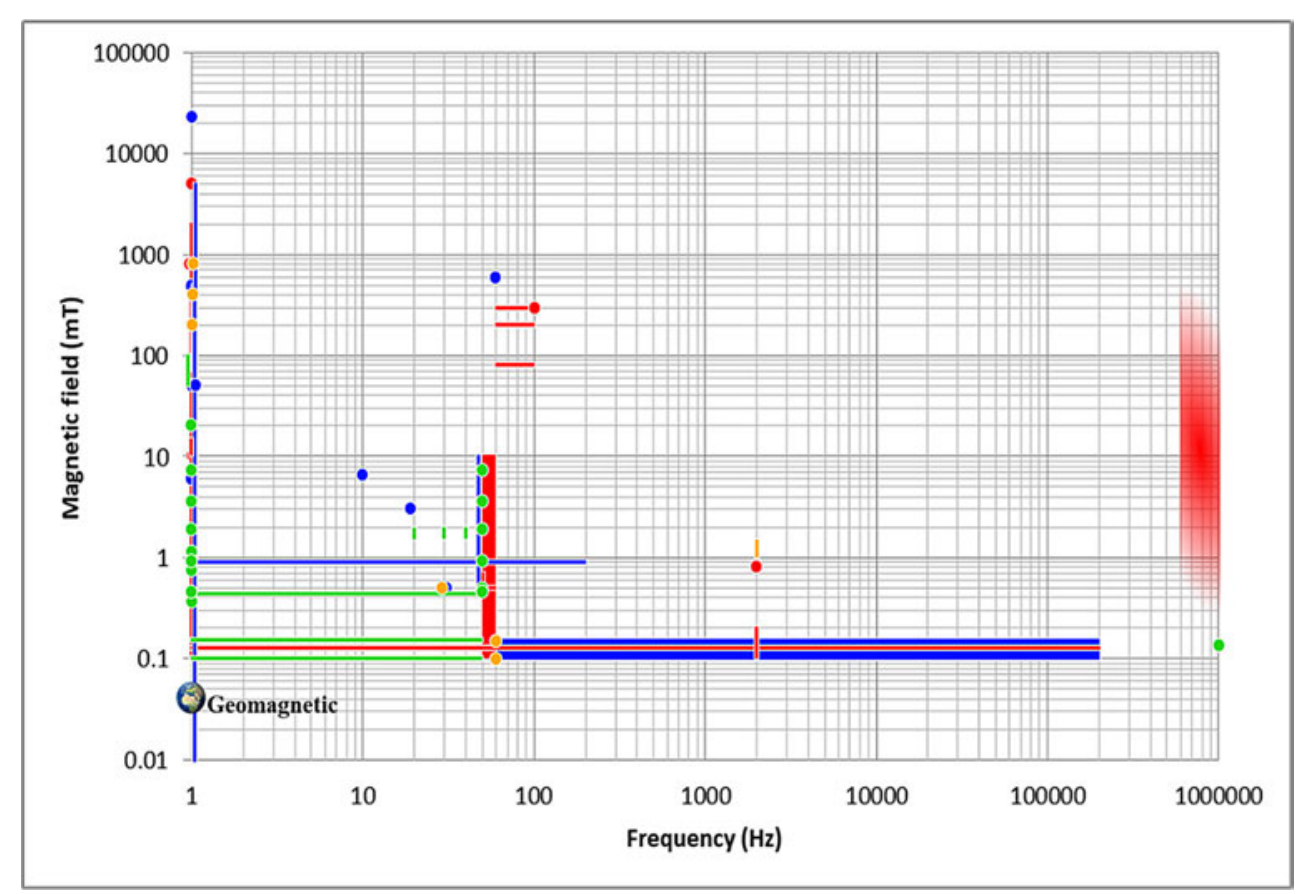


surprisingly, they achieved shorter freezing plateaux and total freezing times than in control experiments with no SMF application. Moreover, there exist a number of interesting papers that, although not focused on freezing kinetics, study the effects of SMFs on cryopreservation of biological specimens. In these experiments, relatively strong SMFs of 200, 400, and $800 \mathrm{mT}$ were successfully employed to increase the survival rate of erythrocytes [27, 28] and dental pulp stem cells [29]. The authors suggested that these benefits are not due to a direct effect of SMFs on water molecules, but on phospholipids that exhibit a highly diamagnetic anisotropic susceptibility. According to the authors, the phospholipids in the cell membranes could be oriented by the torque of the SMF force and this reorientation could reduce the membrane fluidity and the dehydration damage during freezing.

\section{Oscillating EM Fields: the Role of Frequencies in EM Freezing}

Oscillating electric and magnetic fields are defined not only by the field strength, but also by frequency. As a starting point to analyze the importance of frequency in EM freezing, it is convenient to consider the physical behavior of the electromagnetic field. It can be described by the relations between the electric field, $\vec{E}$, and the magnetic field, $\vec{B}$, known as Maxwell's equations:

$$
\begin{aligned}
& \nabla \cdot \vec{E}=\frac{\rho}{\varepsilon_{0}} \\
& \nabla \cdot \vec{B}=0 \\
& \nabla \times \vec{E}=-\frac{\partial \vec{B}}{\partial t} \\
& \nabla \times \vec{B}=\mu_{0} \vec{J}+\mu_{0} \varepsilon_{0} \frac{\partial \vec{E}}{\partial t}
\end{aligned}
$$

where $\rho$ is the charge density $\left(\mathrm{C} / \mathrm{m}^{3}\right), \vec{J}$ is the current density $\left(\mathrm{A} / \mathrm{m}^{2}\right), \varepsilon_{0}$ is the permittivity of the vacuum $\left(8.854 \cdot 10^{12} \mathrm{~F} /\right.$ $\mathrm{m})$, and $\mu_{0}$ is the permeability of the vacuum $\left(4 \pi \cdot 10{ }^{7} \mathrm{H} / \mathrm{m}\right)$. Permittivity and permeability are physical variables that describe how an electric or a magnetic field affects and is affected by a medium, respectively. For a given material, its absolute permittivity, $\varepsilon$, and permeability, $\mu$, are related to those of the vacuum by means of its relative permittivity, $\varepsilon_{\mathrm{r}}$, and relative permeability, $\mu_{\mathrm{r}}$, through the equations:

$$
\begin{aligned}
& \varepsilon=\varepsilon_{0} \cdot \varepsilon_{r} \\
& \mu=\mu_{0} \cdot \mu_{r}
\end{aligned}
$$

In the presence of a variable $\mathrm{EF}$, the dielectric response of materials depends on the field frequency and there exist phenomena of dipole relaxation and resonance at different frequencies. This frequency-dependent behavior can be expressed in terms of the complex relative permittivity, $\varepsilon_{\mathrm{r}}{ }^{*}$ :

$\varepsilon_{r}^{*}=\varepsilon_{r}^{\prime}-j \varepsilon_{r}^{\prime \prime}$

where $\varepsilon_{\mathrm{r}}{ }^{\prime}$ is the relative dielectric constant and $\varepsilon_{\mathrm{r}}{ }^{\prime \prime}$ is the loss factor, representing the capacities of the material for storing and dissipating energy, respectively. In polar substances of small molecular size, as water, it can be demonstrated that the frequency dependence of $\varepsilon_{\mathrm{r}}{ }^{\prime}$ and $\varepsilon_{\mathrm{r}}{ }^{\prime \prime}$ satisfies the Debyetype expressions [44]:

$\varepsilon_{r}^{\prime}(\omega)=\varepsilon_{\infty}+\frac{\left(\varepsilon_{s}-\varepsilon_{\infty}\right)}{1+\omega^{2} \tau^{2}}$

$\varepsilon_{r}^{\prime \prime}(\omega)=\frac{\left(\varepsilon_{s}-\varepsilon_{\infty}\right) \omega \tau}{1+\omega^{2} \tau^{2}}$

where $\varepsilon_{\mathrm{s}}$ and $\varepsilon_{\infty}$ are the permittivity values at low and high frequencies, respectively, $\tau$ is the characteristic relaxation time, and $\omega$ is the angular frequency. It should be noted that the angular frequency is measured in $\mathrm{rad} / \mathrm{s}$ in contrast to the EM frequency, f, which is measured in $\mathrm{Hz}$, i.e., cycles/s.

An approximate representation of the real and imaginary parts of the complex permittivity for water at $25^{\circ} \mathrm{C}$ and 1 bar is shown in Fig. 2, calculated from the available values of $\tau$ [8], $\varepsilon_{\mathrm{S}}$ [16], and $\varepsilon_{\infty}$ [4]. The reduction of the real part of $\varepsilon_{\mathrm{r}}$ on reaching high frequencies is due to the fact that polar molecules, whose dipole moments align along EF direction, become unable to follow those field variations on reaching certain frequencies. Therefore, there is a lag between them which increases losses. Then, the maximum of $\varepsilon_{\mathrm{r}}{ }^{\prime \prime}$ and, consequently, the maximum losses appear at the frequency in which $\varepsilon_{\mathrm{r}}{ }^{\prime}$ decreases down to the middle value between $\varepsilon_{\mathrm{s}}$ and $\varepsilon_{\infty}$. The angular frequency at this point is equal to the inverse of the relaxation time of the medium $(\omega=1 / \tau)$. For a given material, temperature is one of the main factors that cause variations in the relaxation angular frequency, the maximum of $\varepsilon_{\mathrm{r}}{ }^{\prime \prime}$ and in $\varepsilon_{\mathrm{s}}$. However, the drastic drop in the mobility of molecules produced by freezing originates a comparatively much bigger

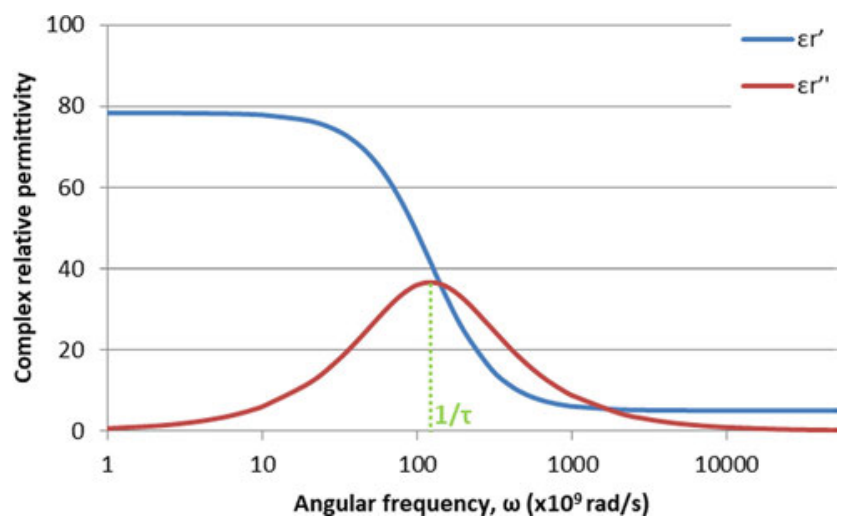

Fig. 2 Debye's model of the complex relative permittivity of water at $25^{\circ} \mathrm{C}$ and 1 bar. Maximum losses appear when angular frequency, $\omega$, equals the inverse of the relaxation time, $\tau$ 
reduction in the frequency of appearance of maximum losses. In this sense, Artemov and Volkov [4] compared the complex relative permittivity of water and ice at $0{ }^{\circ} \mathrm{C}$. The corresponding graphs were very similar, but the angular frequencies of the peak of $\varepsilon_{\mathrm{r}}$ " were shifted six orders of magnitude and, thus, the relaxation times were $1.7 \cdot 10{ }^{11}$ and $2.2 \cdot 10{ }^{5} \mathrm{~s}$ for water and ice, respectively. This provokes, for instance, a much higher absorption of energy by liquid water than by ice in microwave ovens (usually working at $2.45 \mathrm{GHz}$ ). Thus, the ratio of water-to-ice EM wave absorption per unit length is 71.4 at that frequency [13]. So, given the displacement of around six decades in the graph of ice dielectric response versus frequency, it could be reasonable to think that, if interesting effects of EM freezing are sought, frequencies in the order of $\mathrm{kHz}$ should be explored. Considering again the operation of microwave ovens, it is advisable to mention that dipolar polarization is not the only mechanism able to produce heat losses but also ionic conduction, if dissolved charged particles are present, and interfacial polarization, which is a combination of both mechanisms and occurs when a conductive material is dispersed in a non-conductive medium $[3,6]$. Therefore, if significant effects on freezing were observed when applying EF frequencies of several $\mathrm{kHz}$, as suggested above for ice, similar mechanisms to those involved in microwave heating could be proposed as a possible explanation of the action of those fields on freezing.

\section{Application of Oscillating Electric Fields for Freezing: Effects of Frequency on Freezing Kinetics}

There exist in the literature a number of papers focused on the potential effects of the frequency of OEF on freezing kinetics and/or the quality of frozen products. Thus, Sun et al. [55] froze $0.9 \% \mathrm{~K}_{2} \mathrm{MnO}_{4}$ solutions in a $500-\mathrm{V} / \mathrm{m}$ OEF at several frequencies between 1 and $200 \mathrm{kHz}$. From the lowest frequency on, they observed a gradual reduction of the ice crystal size and of the total freezing time and, at $50 \mathrm{kHz}$, a minimum was reached for both parameters. For higher frequencies, both the ice crystal size and the freezing time rose again. The authors remarked that this frequency was very close to $48 \mathrm{kHz}$, where the dielectric relaxation peak of salty ice appears [67]. It should be noted that this value differs from those stated for pure ice in the literature. Thus, at $0{ }^{\circ} \mathrm{C}$, Artemov and Volkov [4] reported a value of $7.23 \mathrm{kHz}$ while, at $-1.15{ }^{\circ} \mathrm{C}$, Popov et al. [46] reported a frequency of the relaxation peak around $5.31 \mathrm{kHz}$. This decrease of the relaxation frequency with temperature may be due to the well-known increase of the dielectric relaxation time of water and ice when decreasing temperatures $[5,51]$.

Similar results were obtained by Mok et al. [33] who froze $0.9 \% \mathrm{NaCl}$ solutions at $-20^{\circ} \mathrm{C}$ while applying pulsed EFs of $178 \mathrm{~V} / \mathrm{m}$ and $50 \%$ duty cycle at different frequencies (1-
$20 \mathrm{kHz}$ ). As observed by Sun et al. [55], these authors detected a reduction of the phase transition time with increasing frequencies. Moreover, at $20 \mathrm{kHz}$, ice crystals were also smaller and rounder than in control samples with no EF application. Mok et al. [34] also applied pulsed EFs at $20 \mathrm{kHz}$ to chicken breasts, but following a time sequence of different duty cycles, namely 0.8 (300 s), $0.5(120 \mathrm{~s})$, and 0.2 (90 s), and applying simultaneously a $1-\mathrm{Hz}$ oscillating magnetic field (OMF) during the third stage of the sequence. The authors managed to keep chicken breasts in a supercooled state at $-6.5{ }^{\circ} \mathrm{C}$ for $12 \mathrm{~h}$, whereas the breasts were partially frozen at that temperature when not subjected to any EM treatment.

Studies at considerably higher frequencies also gave promising results. Thus, Ma et al. [31] froze $0.9 \% \mathrm{NaCl}$ solutions in the presence of several OEFs of strengths between $12.5 \cdot 10^{3}$ and $10^{5} \mathrm{~V} / \mathrm{m}$ and frequencies ranging from $100 \mathrm{kHz}$ to $10 \mathrm{MHz}$. When the samples were subjected to OEFs, a reduction of the ice grain sizes and the associated ice crystallization fraction was observed and the best results were obtained at $10^{5} \mathrm{~V} / \mathrm{m}$ and $1 \mathrm{MHz}$. The authors stated that this frequency was in the region where the complex electric modulus $\mathrm{M}^{*}$ (the inverse of $\varepsilon_{\mathrm{r}}{ }^{*}$ ) of the solution at $-5{ }^{\circ} \mathrm{C}$ shows dielectric relaxation and this could be related to the reduction of the ice crystal size. However, it is important to note that these positive results may be due not just to the employed frequencies but also to the higher OEF strength compared to those of the other two considered works.

\section{Application of Oscillating Magnetic Fields for Freezing: Effects of Frequency on Freezing Kinetics}

Experiments described in the literature to analyze the potential effects of OMF frequency on freezing kinetics have been performed using both commercial equipment and lab prototypes. Commercial equipment allows the study of a limited range of strengths and frequencies but, fortunately, lab prototypes have been developed to extend the range of frequencies under study from 0 to $200 \mathrm{kHz}$ (Fig. 1).

Patented devices and commercial equipment frequently combine static and oscillating magnetic fields and this makes it difficult to discern the specific effects that each of them can have on freezing kinetics. Moreover, it is interesting to note that the range of MF strengths and frequencies covered by patents is considerably wider than that observed in commercial equipment. Thus, the SMF strengths covered by ABI patents range between $0.1 \mathrm{mT}$ and $2 \mathrm{~T}$ [40-42], while values observed in commercial equipment do not exceed $0.22 \mathrm{mT}$ at the center of the central tray in the freezing cabinet [38]. Regarding the OMF, ABI patents cover strength and frequency values of $0.1-100 \mathrm{mT}$ and $50-60 \mathrm{~Hz}$, respectively. Commercial equipment usually has a control panel to select a "CAS energy" percentage, from 0 to $100 \%$, which 
corresponds to different values of OMF strength and frequency. However, the correspondence between "CAS energy" percentages and real OMF strength and frequency values is not provided by the manufacturer and, therefore, the user/food processor has no knowledge of the magnetic fields actually applied. To avoid this inconvenience, several authors in the scientific literature have performed experimental measurements of the real OMF strengths and frequencies for different "CAS energy" conditions in ABI freezers. Even though the results obtained by different authors were not identical, probably because different models of freezer were tested, data show that OMF strength at the center of the freezing cabinet was, in no case, larger than $2 \mathrm{mT}$, while frequency values varied from 0 to $60 \mathrm{~Hz}[11,19,38,47,64]$.

The studies published in the literature show that, at relatively low OMF strengths $(<10 \mathrm{mT})$, the frequency of the mains $(50-60 \mathrm{~Hz})$ or lower seems not to have any effect on supercooling of pure water or saline solutions. Thus, Watanabe et al. [59] froze pure water and $1 \mathrm{~mol} / \mathrm{kg} \mathrm{NaCl}$ solutions subjected to OMFs of $0.5-10 \mathrm{mT}$ at $50 \mathrm{~Hz}$ and they did not observe any influence of the OMF on supercooling. Similar results were obtained by Naito et al. [35] when freezing both distilled and saline water under a $0.5-\mathrm{mT}$ OMF at $30 \mathrm{~Hz}$. The same occurred when freezing real foods. Thus, Suzuki et al. [56] and Watanabe et al. [59] froze radish, tuna, sweet potato, and yellow tail fish in a $0.5-\mathrm{mT}$ OMF at $50 \mathrm{~Hz}$ and did not observe any effect of the magnetic field on freezing kinetics, ice crystals, and quality of the products.

These results, obtained in lab prototypes, agree with those found by some authors in commercial ABI CAS freezers. Thus, no effects or few non-reproducible differences were found when freezing garlic [19], pork loin [49], crab sticks [38], Atlantic cod [11], or potato and apple samples [47] in ABI CAS freezers, employing OMF strengths between $0.027 \mathrm{mT}$ [11] and $1.95 \mathrm{mT}$ [38] and frequencies ranging from 10 up to $58 \mathrm{~Hz}$. However, some positive results of ABI CAS freezers have also been reported in the literature. Thus, Yamamoto et al. [64] froze chicken breasts in a CAS freezer with OMF strengths between 1.5 and $2 \mathrm{mT}$ and frequencies of 20, 30, and $40 \mathrm{~Hz}$. Even though after 1 week of storage, no difference in quality was found by comparing CAS experiments with chicken samples frozen by conventional methods, after 6-month storage, rupture stress was significantly increased only in the samples frozen without CAS. Moreover, Iwasaka et al. [18] froze DMEM culture medium and $\mathrm{NaCl}$ solutions in two CAS freezers that generated pulsed MFs of $6.5 \mathrm{mT}$ and $3 \mathrm{mT}$ at 10 and $18 \mathrm{~Hz}$, respectively, and observed differences in the scattered light. The results seem to indicate a reduction in the ice crystal sizes of the solutions frozen with pulsed MF. In addition, there are a number of works using CAS equipment, with OMF frequencies $\leq 60 \mathrm{~Hz}$, which claim to improve cell cryopreservation. For example, Kaku et al. [22] found higher survival rate in human periodontal ligament cells, frozen in a CAS freezer at $0.1 \mathrm{mT} / 60 \mathrm{~Hz}$, than in those frozen with no OMF application. Similar results were described by Kojima et al. [25] and Koseki et al. [26] who froze rat mesenchymal stem cells and mouse osteoblasts, respectively, at $0.1 \mathrm{mT}$ and observed that, compared to control samples frozen with no OMF application, the cell survival and proliferation rate increased after thawing.

Some results, obtained in lab prototypes, seem to indicate that frequencies higher than that of the mains could significantly improve freezing kinetics. Thus, Mihara et al. [32] and Niino et al. [36] froze physiological saline solutions with and without a $0.12 \pm 0.02-\mathrm{mT}$ MF at different frequencies from $50 \mathrm{~Hz}$ up to $200 \mathrm{kHz}$. At $50 \mathrm{~Hz}$, they did not find significant differences compared with the control, but they observed that supercooling increased with frequency and detected a maximum (close to $20{ }^{\circ} \mathrm{C}$ ) at $2 \mathrm{kHz}$. Semikhina and Kiselev [52] also observed an effect of frequency when freezing bidistilled water previously subjected to OMFs of $0.88 \mathrm{mT}$ and $10^{2}-200 \mathrm{~Hz}$ for $5 \mathrm{~h}$. The authors reported an increase in supercooling due to the MF exposition and they stated that the supercooling extent depended both on the OMF strength and frequency. Unfortunately, to the best of our knowledge, additional papers by other authors that confirm these results do not exist in the literature.

\section{Oscillating Magnetic Fields as a Means to Generate Oscillating Electric Fields}

In the previous sections, OEFs and OMFs have been analyzed as independent fields. However, it is well-known that, according to Faraday's law, (Eq. 3), any variable MF will induce a variable non-conservative $\mathrm{EF}$ whose orientation is such that, if there were a conductor present, the induced current would generate a MF which would tend to oppose the variation of MF flux (as stated in Lenz's law). This opens the possibility to generate OEFs in a wide range of frequencies by means of OMFs, as announced in Jha et al. [20].

As previously commented, the results obtained by Mok et al. [33] and Sun et al. [55] suggest that relatively weak OEFs can improve freezing kinetics if applied at an adequate frequency. These OEFs could be induced in the range of frequencies of interest by means of OMFs of the same frequencies and, therefore, the common problems associated to OEF generators would be avoided.

To illustrate how to quantify the strength of an induced EF, let us suppose a variable $\mathrm{MF}, \overrightarrow{\mathrm{B}}$, confined in a circular region of radius $R$ (Fig. 3). Then, from the circulation of the induced EF along a circular concentric path of radius $r$, the value of the EF strength is easily calculated, being: 


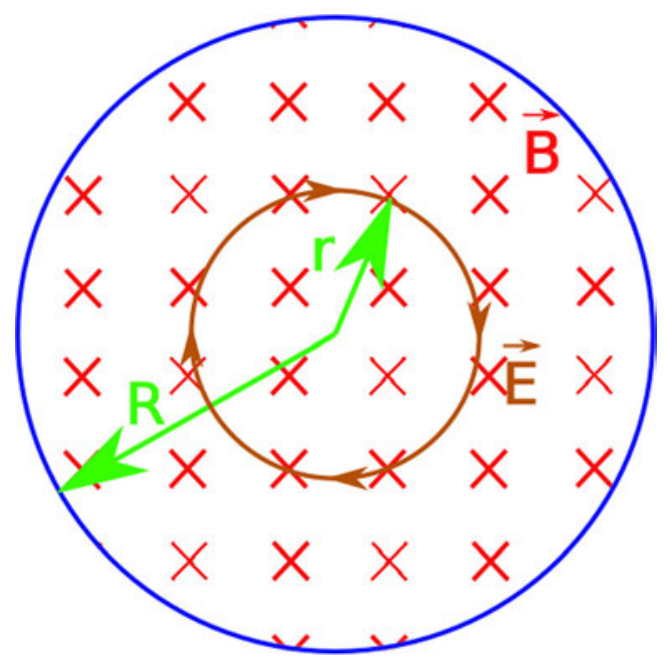

Fig. 3 Electric field induced by a variation of magnetic flux

$|\overrightarrow{\mathrm{E}}|=\frac{r}{2} \frac{\mathrm{d}|\overrightarrow{\mathrm{B}}|}{\mathrm{dt}}$

when $r \leq R$, whereas for $r>R$ it is:

$|\overrightarrow{\mathrm{E}}|=\frac{R^{2} \mathrm{~d}}{2 r} \frac{|\overrightarrow{\mathrm{B}}|}{\mathrm{dt}}$

Supposing that the MF is instantaneously uniform on the circle surface and it is only time-varying in a sinusoidal fashion at a constant frequency $\mathrm{f}$ :

$|\overrightarrow{\mathrm{B}}|=\mathrm{B}_{\mathrm{pk}} \cdot \sin (2 \pi \mathrm{ft})$

then, taking into account that in this case there is a maximum of the induced EF strength for $r=R$, this is:

$|\overrightarrow{\mathrm{E}}|_{\max }=\frac{R}{2} \cdot \mathrm{B}_{\mathrm{pk}} \cdot 2 \pi \mathrm{f} \cdot \cos (2 \pi \mathrm{ft})$

As an example, Rodríguez [48] designed and built a cylindrical $(5.75 \mathrm{~cm}$ radio and $8.6 \mathrm{~cm}$ high) air core $\mathrm{OMF}$ generator for laboratory experiments. The device consisted of a solenoid of 75 turns distributed in one layer around a lab-built bobbin and an electronic inverter. For the reduction of problems derived from the high frequency, Litz wire was employed to construct the solenoid. In turn, the inverter was able to work in a wide range of frequencies between $1 \mathrm{~Hz}$ and $50 \mathrm{kHz}$, thanks to adequate strategies for the driving of switches at high and low frequencies. The drive of the switches was carried out by a commercial microcontroller unit. In this equipment, the sample inside the coil could be subjected to a peak OMF strength of, at least, $1.13 \mathrm{mT}$, i.e., a root mean square (RMS) value of $0.8 \mathrm{mT}$, which is near the value used in commercial CAS equipment [38]. According to (Eq. 13), at
$50 \mathrm{kHz}$, OMF strength gives rise to an OEF RMS value of $7.2 \mathrm{~V} / \mathrm{m}$.

Experimentation with this device is currently in process to test if, as observed by Mok et al. [33] and Sun et al. [55], frequency, up to $50 \mathrm{kHz}$, can affect freezing kinetics of pure water and aqueous solutions at this weak OEF strength. If effective, the employment of OMF generators to produce OEFs, able to increase supercooling, would result in an important technological advance because they are much more manageable than electrodes. Moreover, the scale-up of the described design to fit industrial conditions would not be any significant technological hindrance [48].

\section{Conclusion}

Although in the last years an increasing number of scientific papers on the effect of both static and oscillating electric and magnetic fields on freezing have been published, nowadays, there is still no clear evidence of their efficacy in improving freezing kinetics and/or the quality of frozen products.

To shed light on this subject, systematic experimentation is needed. Well-defined experiments that can be replicated and confirmed by different laboratories should be performed. When looking for the effects of EMFs on supercooling, the number of replicated experiments should be enough to capture the stochastic nature of ice nucleation. Moreover, when working with biological materials, their inherent variability should be considered. Thus, to draw valid conclusions, special care should be taken in the experimental design and the subsequent statistical analysis of the data.

When assessing the effects of static electric or magnetic fields on freezing, critical values in a wide strength range should be investigated. To do so, all the factors that can play a role in the observed results should be clearly identified and considered. Among them, special attention should be paid to the sample composition and size, the presence of impurities, the temperature, the cooling rate, and the spatial electric and magnetic gradients that can be established throughout the sample, among others. Experimentation should be first performed in the simplest matrix, that is, in pure water and, then, the effects of EMFs on other molecules with potential impact on freezing kinetics and/or the quality of frozen foods should also be analyzed in depth. Only after finding positive results, mechanisms involved in such results should be investigated.

When studying oscillating electric and magnetic fields, both critical strength and frequency values, and also any potential interrelation between them, should be sought. Discerning the separate effects of oscillating electric and magnetic fields on freezing seems not to be simple because, according to Ampère-Maxwell law and Faraday's law, the presence of an oscillating either electric or magnetic field successively produces an induced oscillating magnetic or electric 
field, respectively. Obviously, the character of the field source (magnetic or electric) determines the main field, which is much stronger than the induced one.

The role of frequency and dielectric relaxation on water supercooling should be analyzed in depth because it opens a door to explain the potential effects of OMFs on freezing. If significant effects of weak OEFs at specific frequencies on supercooling were confirmed, critical values to achieve the pursued effects should be identified. Future research should also clarify the potential effect of different solutes and temperatures.

Moreover, lab prototypes, able to produce wide OMF strength and frequency ranges, are needed. The OEFs induced in these devices, at different OMF strength and frequency conditions, should be calculated to consider their potential contribution to any effect observed. If the effects of OMF freezers on supercooling and freezing kinetics were proved at some OMF strength or frequency and, actually, assigned to the induced OEF, physics underlying OMF freezing could be finally explained and the successful industrial implementation of electromagnetic freezing could be a relatively easy task. Thus, the production of OMF freezers, able to induce OEFs with critical strength and frequency values on supercooling, would be a relatively simple solution.

Funding Information This work has been supported by the Spanish MINECO through the Project AGL2012 39756 C02 01. A.C. Rodríguez is supported by the BES 2013065942 pre doctoral grant, also from MINECO.

\section{References}

1. ABI Co., L. (2007) CAS: cells alive system. The CAS energy function has an international patent

2. Aleksandrov VD, Barannikov AA, Dobritsa NV (2000) Effect of magnetic field on the supercooling of water drops. Inorg Mater 36(9):895 898. https://doi.org/10.1007/bf02758700

3. Anwar J, Shafique U, Rehman R, Salman M, Dar A, Anzano JM et al (2011) Microwave chemistry: effect of ions on dielectric heating in microwave ovens. Arab J Chem 8(1):100 104

4. Artemov VG, Volkov AA (2014) Water and ice dielectric spectra scaling at 0 degrees C. Ferroelectrics 466(1):158 165. https://doi. org/10.1080/00150193.2014.895216

5. Artemov VG, Ryzhkin IA, Sinitsyn VV (2015) Similarity of the dielectric relaxation processes and transport characteristics in water and ice. JETP Lett 102(1):41 45. https://doi.org/10.1134/ S0021364015130020

6. Barba AA, d'Amore M (2012) Relevance of dielectric properties in microwave assisted processes. In: Microwave Materials Characterization. InTech

7. Burke MJ, George MF, Bryant RG (1975) Water in plant tissues and frost hardiness. In: Press NYA (ed) Water relations of foods. Food science and technology monographs. R. B. Duckworth, pp 111135
8. Chaplin M (2017) Water structure and science. Retrieved from http://www1.1sbu.ac.uk/water/water_structure_science.html. Accessed 21 Jan 2019

9. Dalvi Isfahan M, Hamdami N, Le Bail A (2016) Effect of freezing under electrostatic field on the quality of lamb meat. Innovative Food Sci Emerg Technol 37:68 73. https://doi.org/10.1016/j.ifset. 2016.07.028

10. Dalvi Isfahan M, Hamdami N, Xanthakis E, Le Bail A (2017) Review on the control of ice nucleation by ultrasound waves, elec tric and magnetic fields. J Food Eng 195:222 234. https://doi.org/ 10.1016/j.jfoodeng.2016.10.001

11. Erikson U, Kjørsvik E, Bardal T, Digre H, Schei M, Søreide TS, Aursand IG (2016) Quality of Atlantic cod frozen in cell alive system, air blast, and cold storage freezers. J Aquat Food Prod Technol 25:1 20. https://doi.org/10.1080/10498850.2015.1007542

12. Fikiin K (2009) Emerging and novel freezing processes. In: Frozen Food Science and Technology, pp 101123

13. Hales A, Quarini G, Hilton G, Ash D, Lucas E, McBryde D, Yun X (2014) Ice fraction measurement of ice slurries through electromag netic attenuation. Int J Refrig 47(0):98 104. https://doi.org/10. 1016/j.ijrefrig.2014.06.004

14. Hozumi T, Saito A, Okawa S, Watanabe K (2003) Effects of elec trode materials on freezing of supercooled water in electric freeze control. Int J Refrig 26(5):537 542. https://doi.org/10.1016/S0140 7007(03)00008 2

15. Hozumi T, Saito A, Okawa S, Eshita Y (2005) Effects of shapes of electrodes on freezing of supercooled water in electric freeze con trol. Int J Refrig 28(3):389 395. https://doi.org/10.1016/j.ijrefrig. 2004.08.009

16. IAPWS (2011) Revised supplementary release on properties of liq uid water at $0.1 \mathrm{MPa}$. Retrieved from http://www.iapws.org/ relguide/LiquidWater.pdf. Accessed 21 Jan 2019

17. IFP Ltd. (2015) Proton freezer catalog. Retrieved from http://ifp ltd.co.jp/img/proton freezer catalog en.pdf. Accessed 21 Jan 2019

18. Iwasaka M, Onishi M, Kurita S, Owada N (2011) Effects of pulsed magnetic fields on the light scattering property of the freezing pro cess of aqueous solutions. J Appl Phys 109(7):07E320 07E323

19. James C, Reitz B, James S (2015) The freezing characteristics of garlic bulbs (Allium sativum L.) frozen conventionally or with the assistance of an oscillating weak magnetic field. Food Bioprocess Technol 8(3):702 708

20. Jha PK, Xanthakis E, Jury V, Le Bail A (2017) An overview on magnetic field and electric field interactions with ice crystallisation; application in the case of frozen food. Crystals 7(10). https://doi. org/10 3390/cryst7100299

21. Jia G, He X, Nirasawa S, Tatsumi E, Liu H, Liu H (2017) Effects of high voltage electrostatic field on the freezing behavior and quality of pork tenderloin. J Food Eng 204:18 26. https://doi.org/10.1016/ j.jfoodeng.2017.01.020

22. Kaku M, Kamada H, Kawata T, Koseki H, Abedini S, Kojima S, Tanne K (2010) Cryopreservation of periodontal ligament cells with magnetic field for tooth banking. Cryobiology 61(1):73 78. https:// doi.org/10.1016/j.cryobiol.2010.05.003

23. Kelly T (2008) Mr. Freeze. Forbes

24. Kim SC, Shin JM, Lee SW, Kim CH, Kwon YC, Son KY (2007) WO2007094556 A2

25. Kojima S, Kaku M, Kawata T, Sumi H, Shikata H, Abonti TR et al (2013) Cryopreservation of rat MSCs by use of a programmed freezer with magnetic field. Cryobiology 67(3):258 263. https:// doi.org/10.1016/j.cryobiol.2013.08.003

26. Koseki H, Kaku M, Kawata T, Kojima S, Sumi H, Shikata H et al (2013) Cryopreservation of osteoblasts by use of a programmed freezer with a magnetic field. Cryoletters 34(1):10 19

27. Lin C Y, Chang W J, Lee S Y, Feng S W, Lin C T, Fan K S, Huang H M (2013a) Influence of a static magnetic field on the slow 
freezing of human erythrocytes. Int J Radiat Biol 89(1):51 56. https://doi.org/10.3109/09553002.2012.717731

28. Lin C Y, Wei P L, Chang W J, Huang Y K, Feng S W, Lin C T, Huang H M (2013b) Slow freezing coupled static magnetic field exposure enhances cryopreservative efficiency a study on human erythrocytes. PLoS One 8(3):e58988. https://doi.org/10.1371/ journal.pone. 0058988

29. Lin SL, Chang WJ, Lin CY, Hsieh SC, Lee SY, Fan KH et al (2014) Static magnetic field increases survival rate of dental pulp stem cells during DMSO free cryopreservation. Electromagn Biol Med 34: 302 308. https://doi.org/10.3109/15368378.2014.919588

30. Lou Y J, Zhao H X, Li W B, Han J T (2013) Experimental of the effects of static magnetic field on carp frozen process. J Shandong Univ, Eng Sci 43(6):89 95

31. Ma Y, Zhong L, Gao J, Liu L, Hu H, Yu Q (2013) Manipulating ice crystallization of 0.9 wt.\% $\mathrm{NaCl}$ aqueous solution by alternating current electric field. Appl Phys Lett 102(18):183701. https://doi. org/10.1063/1.4804287

32. Mihara M, Nakagawa T, Noguchi S, Dohi T, Masamune K, Niino T, Yamashita H (2012) EP Patent 2499924 A1

33. Mok JH, Choi W, Park SH, Lee SH, Jun S (2015) Emerging pulsed electric field (PEF) and static magnetic field (SMF) combination technology for food freezing. Int J Refrig 50:137 145. https://doi. org/10.1016/j.ijrefrig.2014.10.025

34. Mok JH, Her J Y, Kang T, Hoptowit R, Jun S (2017) Effects of pulsed electric field (PEF) and oscillating magnetic field (OMF) combination technology on the extension of supercooling for chick en breasts. J Food Eng 196:27 35. https://doi.org/10.1016/j. jfoodeng.2016.10.002

35. Naito M, Hirai S, Mihara M, Terayama H, Hatayama N, Hayashi S et al (2012) Effect of a magnetic field on drosophila under supercooled conditions. PLoS One 7(12):e51902. https://doi.org/ 10.1371/journal.pone.0051902

36. Niino T, Nakagawa T, Noguchi S, Sato I, Kawai T, Yamashita H et al (2012) Whole ovary cryopreservation applying supercooling under magnetic field: mechanical engineering* organ cryopreserva tion* reproductive technique. Academic Collaborations for Sick Children 5(1):14 20. https://doi.org/10.5108/acsc.5.14

37. Otero L, Rodríguez AC, Pérez Mateos M, Sanz PD (2016) Effects of magnetic fields on freezing: application to biological products. Compr Rev Food Sci Food Saf 15(3):646 667

38. Otero L, Pérez Mateos M, Rodríguez AC, Sanz PD (2017) Electromagnetic freezing: effects of weak oscillating magnetic fields on crab sticks. J Food Eng 200:87 94. https://doi.org/10. 1016/j.jfoodeng.2016.12.018

39. Otero L, Rodríguez AC, Sanz PD (2018) Effects of static magnetic fields on supercooling and freezing kinetics of pure water and $0.9 \%$ $\mathrm{NaCl}$ solutions. J Food Eng 217(Supplement C):34 42. https://doi. org/10.1016/j.jfoodeng.2017.08.007

40. Owada N (2007) US Patent No. 7237400

41. Owada N, Kurita S (2001) US Patent No. 6250087

42. Owada N, Saito S (2010) US Patent 7810340B2

43. Petersen A, Schneider H, Rau G, Glasmacher B (2006) A new approach for freezing of aqueous solutions under active control of the nucleation temperature. Cryobiology 53(2):248 257. https:// doi.org/10.1016/j.cryobiol.2006.06.005

44. Pethig R (2017) Dielectric Polarization Dielectrophoresis. Wiley, pp 145166

45. Petzold G, Aguilera JM (2009) Ice morphology: fundamentals and technological applications in foods. Food Biophys 4(Journal Article):378 396

46. Popov I, Puzenko A, Khamzin A, Feldman Y (2015) The dynamic crossover in dielectric relaxation behavior of ice $\mathrm{I}_{\mathrm{h}}$. Phys Chem Chem Phys 17(2):1489 1497. https://doi.org/10.1039/c4cp04271a

47. Purnell G, James C, James SJ (2017) The effects of applying oscil lating magnetic fields during the freezing of apple and potato. Food
Bioprocess Technol 10:2113 2122. https://doi.org/10.1007/ s11947017 19833

48. Rodríguez AC (2017) Characterization of electromagnetic freezing in food matrixes and model food. (Doctoral Thesis), Universidad Politécnica de Madrid, Madrid

49. Rodríguez AC, James C, James SJ (2017) Effects of weak oscillat ing magnetic fields on the freezing of pork loin. Food Bioprocess Technol 10:1615 1621. https://doi.org/10.1007/s11947 0171931 2

50. Ryoho Freeze Systems Co, L. (2017) Proton freezing. Frozen prin ciple/effect. Retrieved from http://www.proton group.net/top/ service/technic.html. Accessed 21 Jan 2019

51. Sasaki K, Kita R, Shinyashiki N, Yagihara S (2016) Dielectric re laxation time of ice Ih with different preparation. J Phys Chem B 120(16):3950 3953. https://doi.org/10.1021/acs.jpcb.6b01218

52. Semikhina LP, Kiselev VF (1988) Effect of weak magnetic fields on the properties of water and ice. Sov Phys J 31(5):351 354. https:// doi.org/10.1007/bf01243721

53. Shevkunov SV, Vegiri A (2002) Electric field induced transitions in water clusters. J Mol Struct THEOCHEM 593(1 3):19 32. https:// doi.org/10.1016/S0166 1280(02)001112

54. Stan CA, Tang SKY, Bishop KJM, Whitesides GM (2011) Externally applied electric fields up to $1.6 \times 10^{5} \mathrm{~V} / \mathrm{m}$ do not affect the homogeneous nucleation of ice in supercooled water. J Phys Chem B 115(5):1089 1097. https://doi.org/10.1021/jp110437x

55. Sun W, Xu X, Sun W, Ying L, Xu C (2006) Effect of alternated electric field on the ice formation during freezing process of $0.9 \% \mathrm{~K}_{2} \mathrm{MnO}_{4}$ water. Icpasm 2005: Proceedings of the 8 th International Conference on Properties and Applications of Dielectric Materials, Vols 1 and 2, 774777

56. Suzuki T, Takeuchi Y, Masuda K, Watanabe M, Shirakashi R, Fukuda Y et al (2009) Experimental investigation of effectiveness of magnetic field on food freezing process. Trans Jpn Soc Refrig Air Cond Eng 26:371 386

57. Vegiri A (2001) A molecular dynamics study of structural transi tions in small water clusters in the presence of an external electric field. J Chem Phys 115(9):4175 4185. https://doi.org/10.1063/1. 1388545

58. Vegiri A (2004) Reorientational relaxation and rotational translational coupling in water clusters in a d.c. external electric field. J Mol Liq 110(1):155 168. https://doi.org/10.1016/j.molliq. 2003.09.011

59. Watanabe M, Kanesaka N, Masuda K, Suzuki T (2011) Effect of oscillating magnetic field on supercooling in food freezing. Paper presented at the The 23rd IIR International Congress of Refrigeration: Refrigeration for sustanaible development. Prague, Czech Republic

60. Wei S, Xiaobin X, Hong Z, Chuanxiang X (2008) Effects of dipole polarization of water molecules on ice formation under an electro static field. Cryobiology 56(1):93 99. https://doi.org/10.1016/j. cryobiol.2007.10.173

61. Woo MW, Mujumdar AS (2010) Effects of electric and magnetic field on freezing and possible relevance in freeze drying. Dry Technol 28(4):433 443. https://doi.org/10.1080/ 07373930903202077

62. Xanthakis E, Havet M, Chevallier S, Abadie J, Le Bail A (2013) Effect of static electric field on ice crystal size reduction during freezing of pork meat. Innovative Food Sci Emerg Technol 20: 115 120. https://doi.org/10.1016/j.ifset.2013.06.011

63. Xanthakis E, Le Bail A, Havet M (2014) Chapter 30 freezing combined with electrical and magnetic disturbances. In: Sun D W (ed) Emerging Technologies for Food Processing, 2nd edn. Academic Press, San Diego, pp 563579

64. Yamamoto N, Tamura S, Matsushita J, Ishimura K (2005) Fracture properties and microstructure of chicken breasts frozen by electro magnetic freezing. J Home Econ Jpn 56(3):141 151 
65. Zaritzky N (2011) Physical chemical principles in freezing. In: Sun DW (ed) Handbook of frozen food processing and packaging, 2nd edn. CRC Press, Boca Raton, pp 337

66. Zhao H, Hu H, Liu S, Han J (2017) Experimental study on freezing of liquids under static magnetic field. Chin J Chem Eng

67. Zhong L, Xu C, Qiu C (1988) Anomalous high permittivity in salty ice a new dielectric phenomenon. Paper presented at the
Proceedings., Second International Conference on Properties and Applications of Dielectric Materials

68. Zhou Z, Zhao H, Han J (2012) Supercooling and crystallization of water under DC magnetic fields. Huagong Xuebao/CIESC Journal 63(5):1405 1408. https://doi.org/10.3969/j.issn.0438 1157.2012. 05.012 